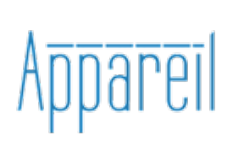

Appareil

$15 \mid 2015$

L'art dans le tout numérique

\title{
L'espace immersif de Monet à Caroline Coppey
}

Jean-Louis Déotte

\section{Q OpenEdition}

Journals

Édition électronique

URL : http://journals.openedition.org/appareil/1746

DOI : 10.4000/appareil. 1746

ISSN : 2101-0714

Éditeur

MSH Paris Nord

Référence électronique

Jean-Louis Déotte, «L'espace immersif de Monet à Caroline Coppey », Appareil [En ligne], 15 | 2015, mis en ligne le 20 juin 2013, consulté le 05 août 2020. URL : http://journals.openedition.org/appareil/ 1746

Ce document a été généré automatiquement le 5 août 2020.

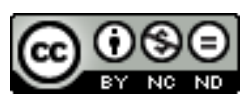

Appareil est mis à disposition selon les termes de la Licence Creative Commons Attribution - Pas d'Utilisation Commerciale - Pas de Modification 4.0 International. 


\title{
L'espace immersif de Monet à Caroline Coppey
}

\author{
Jean-Louis Déotte
}

\section{NOTE DE L'ÉDITEUR}

L'exposition des œuvres de Caroline Coppey a eu lieu du 13 juillet au 15 septembre 2013 à Saint-Rémy de Rouergue, au Moulin des Arts de Saint-Rémy et dans le cadre des Sentiers de l'art de Saint-Rémy. Ce texte de Jean-Louis Déotte est publié dans le catalogue de l'exposition.

Vernissage le 12 juillet 2013

www.atelier-blanc.org

www.caroline-coppey.com

1 Contrairement à ce que l'on entend dire trop souvent, les départements d'art des universités peuvent être des lieux de formation pour de futurs artistes et pas seulement de futurs enseignants. Caroline Coppey a fréquenté celui de Strasbourg et a côtoyé en particulier des membres de ce qui allait devenir le groupe le Faisant. Son directeur de thèse, Claude Gagean, enseignait alors aux côtés de J.-L. Flecniakoska, de G. Roesz et E. Michaud, en collaboration avec des philosophes comme D. Payot, J.-L. Nancy et $\mathrm{Ph}$. Lacoue-Labarthe. On ne sait pas toujours que l'on est né sous une bonne étoile...

2 Tout artiste comme tout écrivain est placé devant le dilemme suivant, surtout depuis l'époque des avant-gardes : pour qui travailler depuis que l'art n'a plus de destination, c'est-à-dire depuis l'époque du musée ? Auparavant, il allait de soi d'exposer comme le fait tout artisan. D'où l'immense succès de la notion d'aura benjaminienne, l'aura des œuvres de culte, destinées à demeurer uniques et quasi invisibles. C'est qu'il y a un risque personnel à exposer, et pour le dire autrement, un changement d'échelle pour une production artistique. Prendre ce risque, ce n'est pas seulement assumer le passage de l'espace privé (l'atelier) à l'espace public (le lieu dédié à l'exposition) où s'expose pour tous le double de l'artiste (ce que les Grecs appelaient daïmon, les Modernes, 
l'inconscient, etc.). Le lieu dans lequel le public va lentement s'approprier l'œuvre (car il n'y a pas de public pour telle œuvre, au moins au début) n'est pas celui de l'exposition en général, lieu totalement absorbé aujourd'hui par la marchandise, mais un lieu spécial où s'interpénètrent le domaine propre de l'artiste et celui des flux et reflux de l'art en mouvement. L'exposition n'est donc pas la scène de la séance du psychanalyste, ni la scène publique (ou politique ou guerrière, chère à Arendt), mais une compénétration réussie des deux. Or cet espace hybride a changé de nature depuis le dernier Monet, celui de l'entre-deux-guerres.

3 L'effet d'une œuvre n'est dès lors plus programmable, sinon elle relèverait de la communication (de la publicité), ce qu'avait fort bien compris Schwitters qui, à peu près à la même époque, travaillait dans les deux domaines parallèlement (la communication/l'art). Dans ce sens, l'artiste ne peut jamais savoir comment le spectateur enchaînera sur ce qu'il lui propose. Si l'enchaînement est nécessaire, il est en même temps improbable. La culture de tel n'étant pas celle de tel autre. Ni l'état psychophysiologique. C'est ce qui rend inutile l'art politique, car il n'y a pas de message univoque transmis à des spectateurs qu'il faudrait éduquer ou émanciper.

4 Soit donc l'artiste n'affronte pas l'exposition, qu'il considère comme une insupportable mise à nu, soit il s'y livre complètement et ne livre au public qu'une valeur marchande (Jeff Koons, Damien Hirst, etc.). Soit il subsiste dans son propre écrin qui lui sert d'étui où quelques pièces collectionnées, venues d'ailleurs, témoignent qu'un autre monde existe à l'extérieur. C'est le particulier qui vit le jour sous Louis-Philippe et qui a reçu une certaine reconnaissance avec l'invention de l'art brut par Dubuffet, art, qui, loin d'être l'art des aliénés est plutôt celui des insulaires les plus radicaux. Soit il l'affronte et n'est même que cela, son agent, celui qui provoque on ne sait quel processus psychophysiologique. C'est ce que Dali, avec une très grande intelligence du marché, avait su formater avec beaucoup de doigté : notre incapacité native à échapper à la fantasmagorie.

Car pour l'humanité, il n'y a que des époques de la fantasmagorie, par exemple les femmes aquatiques et prépubères de l'art nouveau appartenaient bien à une époque, le $\mathrm{XIX}^{\mathrm{e}}$ siècle, qui certes inventa l'industrie, mais ne sut prolonger la nature par la technique.

Caroline Coppey a su répondre à ces deux exigences : celle de l'exposition, celle de l'époque de la technique, et accepter deux contraintes : dès l'atelier, imaginer ce que les esthéticiens nomment la réception, et dans un milieu totalement artificiel, innerver la nature avec des ingrédients chimiques.

Quand l'exposition de l'œuvre est réussie, on peut parler d'osmose. Entre le monde propre de l'artiste et l'anonyme présence d'un public où chaque jugement esthétique vaut pour celui de tout autre, il y a passage de l'un dans l'autre. Ce n'est pas vraiment l'interactivité des artistes du numérique, mais la souplesse de l'espace entre le jeune enfant et sa mère (le play de Winnicott que permet l'espace transitionnel opposé au game, au jeu de règles).

Quand la nature est efficacement innervée par la technique, il ne s'agit pas d'apparier selon le principe du même des éléments hétérogènes, voire des éléments homogènes, mais de rapprocher des éléments semblables, parce que ce qui est au cœur de la nature, c'est la puissance de s'imiter soi-même, ce que les Anciens appelaient mimèsis. En effet, la mimèsis originaire est la seule voie ouverte à l'humanité pour échapper à la fantasmagorie où domine le Même. Dans l'art pariétal, nous rappelle Benjamin, c'est la 
même main qui a fléché l'animal et qui l'a honoré sur la paroi. Entre les deux gestes, s'est imposé le principe du semblable qui a généré une autre forme, à l'image ou à l'imitation de la première. Entre les deux, et cela est fidèle au principe de la mimèsis, il y a eu passage à une puissance supérieure, ce qui fit dire que la représentation révèle en la supplémentant la simple présence. Cette innervation est un geste technique, et suppose donc toute une culture artistique. Ce dont ne dispose pas l'artiste "brut », par définition.

Sans le long travail analytique réalisé pour sa thèse par C. Coppey sur l'œuvre de Monet, la collection des couleurs de la palette impressionniste n'aurait pas été imitée par toujours plus d'abstraction et déployée sans limites. C'est de sa série de Palettes, qu'elle va s'efforcer de toujours enrichir, qu'elle repartira pour ouvrir telle ou telle série, selon des supports différents, des chiffons, des toiles de jute, des bocaux de verre, des peintures grand format voire des supports numériques.

C'est ce programme artistique qui nous retiendra, indissociable de son intention.

Chez elle, fine lectrice des théoriciens du modernisme et en particulier de R. Krauss et de son texte célèbre sur la grille ${ }^{1}$ comme constituant essentiel de l'art moderne, un diagramme a d'entrée de jeu structuré les toiles. Comme le terme de diagramme appartient largement à la philosophie de Deleuze-Guattari et qu'il s'est trouvé pour cette raison surexploité et a perdu peu à peu toute force conceptuelle, je lui préférerai le terme d'appareil. Par ce terme, il faut entendre déjà appareillage, au sens où les maçons appareillent, depuis toujours, un mur. On peut aisément le retrouver avec le sens plus abstrait de structure chez Vitruve. Dans l'édification d'un mur, le choix des matériaux est essentiel. On aura ainsi deux extrêmes: des pierres, provenant de la même veine géologique, taillées strictement de la même manière pour obtenir la régularité d'un appareil classique. Et, à l'autre bord, des matériaux hétérogènes ne présentant pas la régularité, voire la répétition lassante du premier ordre. Entre les deux, toutes les variations furent possibles : de l'appareil en pierres sèches, en passant par l'appareil cyclopéen, l'appareil polygonal, l'appareil régulier, l'appareil moulé, l'appareil à chaînage, etc. L'appareil assure donc la solidité du mur. Il y a peut-être un degré zéro, ou un aboutissement: c'est le béton lisse, où l'on ne distingue plus la structure des matériaux, ce qui nous conduirait à ranger le béton du côté des matières plastiques où la distinction forme/matière se dissout définitivement. Très tôt, avant 1998, dans une proximité à Monet, chez Coppey, les "formes " colorées étaient répétées de telle manière que leur coexistence ordonnée conduisait le plus souvent à la saturation du support. Puis, elles se sont espacées, gagnant en autonomie, puis en singularité, pour se rapprocher de l'état de monade, sachant que chez Leibniz, chaque monade exprime à sa manière le monde.

12 Le terme d'« appareil » a un privilège en français qu'il n'a pas dans les autres langues (" apparat », « machine », " apparatus », etc.) : celui de la polysémie. Ce qui complique la tâche du traducteur, qui doit comme toujours faire un choix entre l'idiomatique et la communication mondialisée. En français donc, bien plus qu'une prothèse (pour un handicap) ou un dispositif (lié au pouvoir comme chez Foucault), l'appareil désigne les superstructures d'un navire, en particulier les voiles et les cordages, d'où l'idée qu'un bateau peut appareiller. C'est ce qui permet le mouvement, le déplacement. C'est aussi ce qui conditionne l'apparaître des choses, en particulier dans l'ordre du rituel. On parlera d'un appareil cérémoniel. Et à partir de là, tout ce qui vient embellir le paraître dans le cadre des rencontres. On s'apercevra alors qu'il n'y a pas de vie publique sans 
ces codes de présentation des uns aux autres, même dans la vie quotidienne. C'est encore l'ensemble des notes et références d'un texte constituant son appareil critique. Dès lors, il faut bien convenir que cet apparat est plus qu'une parure qui viendrait parfaire la nature, mais que tous nos gestes, nos attitudes, nos manières d'être, nos affects, etc., sont appareillés de la matière la plus intime. Il devient de plus en plus problématique de distinguer le laboratoire intime de l'artiste de l'exposition virtuelle de son travail.

Mais si le mouvement de la métaphysique classique a conduit à distinguer puis à figer ce monde de l'apparence opposé au monde de l'être (l'en soi : la substance), jusqu'à ce que l'apparence ne soit plus qu'une sorte d'ornementation (illusoire) des choses, les Anciens ne concevaient pas la Nature de cette manière quand ils parlaient de cosmos. Pour eux, l'ordre du monde apparaissant était beau. Et si l'on devait forger aujourd'hui l'idée d'une cosmétique, c'est cela qu'il faudrait entendre. Mais il nous faudrait accepter en outre l'idée d'une cosmétique générale, parce qu'il y a une pluralité de mondes et à cause de cela des différends cosmétiques majeurs, mais c'est une autre affaire.

Dans sa thèse sur Monet, $C$. Coppey nous rappelle ce qu'était l'apparence du monde sensible pour Monet et sa volonté d'en rendre compte. Sa correspondance, qu'elle étudie, peut nous sembler désuète par ses préoccupations. Il est indéniable qu'entre le monde des impressionnistes et le nôtre, il y a plus qu'un abîme : est-ce que la nature est encore notre « référentiel»? Ne se donne-t-elle pas toujours appareillée, et depuis la Renaissance, par la perspective, la camera obscura, puis au XVIII ${ }^{\mathrm{e}}$ siècle, le musée, au XIX la photographie et le stéréoscope pour un peintre comme Courbet ${ }^{2}$ ? Et finalement par le cinéma?

Ce qui demeure, c'est la logique suivante sur fond de surface de projection : l'appareil artistique que Monet a tenté de mettre en place (la touche, les séries-thématiques, les séries-variation, les motifs-morceaux, etc.) l'a été pour rendre compte de l'organisation interne du sensible, naturel et artificiel (les Cathédrales...). Comme on le sait, il ne s'agissait pas de satisfaire une volonté d'imitation, au sens académique du terme, puisque le dessin n'avait plus vraiment en charge la circonscription de la forme. D'où la rupture avec le disegno qui s'était imposé à la Renaissance. Mais il fallait que la structure de sa peinture soit la plus à même d'approcher celle de l'apparence. Avec la conscience de l'impossibilité finale de la chose et une certaine reconnaissance du sublime sensible sous la forme de la certitude d'une tâche infinie. Ce fut technique (artefact) contre teckhnè (d'apparition). Appareil artistique contre cosmétique «naturelle».

Le jardin de Giverny, cultivé en propre par Monet, fut bien plus qu'une source nouvelle d'inspiration, un microcosme mis à disposition de l'art, mais la mise à l'épreuve des prototypes d'appareil successifs qu'il élaborait en peinture. Plus qu'un projet, moins qu'un programme.

17 On peut distinguer deux aspects dans l'œuvre de C. Coppey à l'épreuve de Monet.

- Un défi que ces artistes ont lancé : ne jamais succomber au Même. Il faut pour cela rester dans l'ordre du semblable à condition qu'une singularité (une touche par exemple) que l'on invente soit semblable à une autre. Ce en quoi consiste leur entreprise paradoxale : sur un même support, placer de semblables couleurs qui seront toutes très petitement dissemblables les unes des autres, par un coloris, par une forme, une grandeur, une matière, un contour, etc., leur donnant à chacune une identité propre. L'ensemble de ces taches, qui s'attirent et se repoussent en même temps, 
formant un champ (c. Coppey parle de plan) que l'on pourrait assimiler à une surface de conflits multiples. Le champ est un terrain de jeu (play).

Il y a là une indéniable mise en relation, qui rend tel champ distinct de tous les autres, telle peinture à la fois proche (c'est un Coppey !) et singulière, car résolvant d'une autre manière le même problème, ce qui implique incontestablement l'existence d'un principe d'individuation pour chaque peinture.

Il y a le risque d'une composition, voire d'une narration, et pourquoi pas, un semblant de petit récit esthétique ? D'autant que dans tel ou tel champ, il y a souvent une tache jaune qui introduit bien un peu de dialectique dans toute cette affaire de couleurs. Mais c'est certainement le prix à payer pour que le jeu ait quelque profondeur ! Et puis ce jeu répond à une recherche inouïe : que mis côte à côte tous ces champs ne fassent qu'un gigantesque arc-en-ciel, sans aucune discontinuité entre les rayons! Ce qui serait rejoindre l'idéal goethéen du Traité des couleurs. Bref, retrouver la lumière pure, mais reconstituée, à travers toute une série de médiations: dès l'abord, la collection des pots, des tissus, des papiers, puis comme seconde étape, la série des Palettes comme modèles réduits, et finalement les grands formats des peintures. On sait que c'est de ce côté-là que penche l'esthétique de Benjamin, en particulier à partir de l'analyse du retable d'Issenheim.

21 - Il n'y a peut-être pas d'histoire de la peinture, au sens d'un progrès technique, mais il y a incontestablement à l'intérieur d'une même époque, si la communication entre les œuvres est avérée, s'il y a donc une contemporanéité effective, un même cadre constitutif que l'on appelle une cosmétique. Alors, les œuvres majeures qui se succèdent, chacune à leur tour, exposent tel ou tel principe constituant de cette cosmétique de telle manière qu'elles feront date. Si ce n'est pas le cas, on reste dans l'ordre du Même. Ce que les artistes partagent depuis le Quattrocento, c'est que la surface d'inscription des signes (picturaux, sculpturaux, théâtraux, architecturaux, littéraires, scientifiques, etc.) est projective. Ce principe est beaucoup plus fondamental que la construction d'un espace homogène selon les lois de la perspective à point de fuite unique. Tout se passe comme si la surface sur laquelle les signes s'inscrivent permettait la projection et l'inscription des traces de ce que l'on voit là-bas, au loin, à travers la vitre transparente, de ce que l'on peut considérer comme dorénavant perdu (la Nature). L'inauguration de la surface comme surface de projection a entraîné ipso facto la désintégration du monde médiéval théologico-politique de l'incarnation (chrétienne). Comme le nouvel art racontait les mêmes histoires que les anciennes images qui illustraient le Texte sacré, les communautés chrétiennes n'ont pas pris immédiatement l'exacte mesure de la révolution qui avait déjà eu lieu (sauf des prédicateurs comme Savonarole), et d'ailleurs les théoriciens de l'art firent comme si de rien n'était (Alberti), le but de la peinture étant prétendument de narrer.

Cette époque de la cosmétique projective a donné lieu à une production inouie et l'on aurait tort de considérer que les différents appareils projectifs qui ont appareillé les arts jusqu'aux années de rupture du XIX siècle n'ont consisté qu'en une mise au pas de l'épanchement de lave libidinale (Lyotard ${ }^{3}$ ) ayant eu lieu à Florence avec Masaccio, Brunelleschi, Alberti. Je ne reprendrai pas ici la remise en cause systématique des constituants de cette cosmétique ${ }^{4}$ avec Manet (l'indécidabilité du sujet de la narration selon Bataille) ou l'opacification de la surface (devenant une membrane quasi biologique chez Cézanne selon Marin) ou l'effacement de la circonscription de la forme chez Monet (Lyotard). En conséquence de quoi, la représentation de l'espace a cessé de 
pouvoir faire advenir la représentation des choses et les signes picturaux, cessant d'être projectifs, ont accaparé toute la destination de la peinture. Et ont perdu leur capacité de valoir pour autre chose qu'eux-mêmes.

D'autant que l'appareil artistique de Monet a prouvé son efficacité en appareillant à nouveaux frais la réalité sensible qui d'effet devint modèle. Entre les Nymphéas et le jardin de Giverny, qui redouble qui ? Cette puissance poétique n'était pas nouvelle, car dès que la perception collective fut appareillée, il s'ensuivit très vite que la « réalité » le devint aussi. Cette réciprocité ou ce jeu de miroirs sont constitutifs de la cosmétique projective : une vue en perspective d'une ville, c'est déjà un projet urbain. Néanmoins, pour tempérer les ardeurs d'urbanistes-concepteurs et de préfets comme Haussmann, il faut rappeler qu'entre les deux, pour que la réalisation du projet soit effective, il faut une large injection de capital ${ }^{5}$ !

Mais entre les Nymphéas et le jardin, il y a une telle action réciproque que la mimèsis pourrait bien s'émanciper de la cosmétique projective : les signes des uns et les réalités de l'autre entrent en correspondance, les spectateurs (car le pluriel est important) de l'Orangerie n'ont plus le loisir de se référer à une réalité potentiellement extérieure à travers un signe pictural qui, malgré sa matérialité, c'est-à-dire son opacité, conserverait encore la capacité de désigner à travers le support.

Est-ce dire que ce monde pictural, sans limites, sans repères, sans articulations, sans interstices et parfaitement englobant pour la masse des spectateurs est inévitablement autoréférentiel? Qu'il prépare l'abstraction lyrique américaine? C'est un monde aquatique, mais à la différence de cette mer peinte par ce peintre chinois de la légende qui, à force de contemplation de son œuvre, s'y noya, rien de tel à l'Orangerie: la possibilité de la contemplation recule car le mouvement de la perception visuelle s'impose, sans directions privilégiées. Ce volume, car c'est une sorte de cavité, ne permet que le balayage perceptif: il s'agit bien d'une expérience immersive, à l'intérieur d'une enveloppe. Faut-il parler alors de signes picturaux immersifs ? L'image de la tactilité vient immédiatement à l'esprit, mais dans l'esthétique de Riegl, elle caractérise plutôt la perception d'un objet proche, opposée à la perception visuelle, offerte aux lointains.

Il serait plus logique de faire l'hypothèse d'une perception de l'intérieur du corps, ou plutôt d'une sensation proprioceptive ou kinesthésique de nos humeurs. On peut suggérer ceci : les arts sont appareillés époque après époque par l'appareil esthétique dominant. Or les Nymphéas sont contemporains du cinéma. Comme l'a montré Benjamin $^{6}$, la perception d'un film est toujours une affaire de masse et, au moins dans un premier temps, la possibilité d'une appropriation collective corporelle. Si nous sommes touchés par un film, c'est qu'il a innervé exceptionnellement notre sensibilité profonde, celle des processus physiologiques comme la tension artérielle, et non parce que nous aurions été hypnotisés ${ }^{7}$ par telle ou telle star. Un film, c'est comme un rêve, mais à condition de penser que ce dernier exprime les processus physiologiques plutôt que des affects inconscients. L'espace des Nymphéas serait donc immersif dans un double sens : aquatique, c'est un espace liquide qui se parcourt avec le sentiment que l'on ne s'y baignera jamais deux fois, intérieur parce que son incorporation est davantage l'affaire de différences de potentiel entre les humeurs que d'inscription de traces mémorielles. Dès lors, la peinture n'a plus comme mère la mémoire. Quels souvenirs conserve-t-on des grands Nymphéas de l'Orangerie? On y baigne dans une atmosphère spéciale, comme si la couche d'air dans laquelle nous vivons faisait de nous 
plutôt des animaux marins que des êtres ailés. C'est exactement la nature du milieu des passages urbains qu'Aragon décrit à la même époque (1926) dans Le Paysan de Paris, à propos du passage de l'Opéra qui connaissait ses dernières années. On sait que cet ouvrage hors normes déclenchera la vive passion de Benjamin pour les passages, lesquels, comme premiers espaces immersifs, préfigurent la structure d'une salle de cinéma.

Or les œuvres numériques ${ }^{8}$ de C. Coppey, comme Palettes 1-300, Accords, et plus particulièrement La Vie en Rose, tenant compte du comportement du spectateur comme de la variation des conditions atmosphériques, de la lumière, de la température, données transmises par internet et provenant d'une station météo, appartiennent à ce milieu de la sensibilité collective, évidemment innervées par la communication qui est devenue notre destin collectif.

On aurait tort à propos des œuvres de C. Coppey de parler de "tableau», tant cette notion dépendante de la décision inouïe, prise par on ne sait qui, au $\mathrm{xv}^{\mathrm{e}}$ siècle à Florence, d'identifier le support de la peinture à une vitre carrée (le "quadro»), a épuisé aujourd'hui toutes ses potentialités. En particulier, et ce n'est pas rien, de nous destiner, nous singularités anonymes, entrant dans ces espaces, à devenir des sujets. L'espace immersif, à la différence du projectif, ne peut plus être structuré par une ligne d'horizon, des points de distance permettant le quadrillage de l'espace de représentation et un point de fuite que l'on appelait dans les traités de perspective, point $d u$ sujet! Dès lors, que l'on ne vienne plus nous parler de crise de la représentation, en particulier politique! Rouillé s'étonne dans la dernière lettre (414, 19 avril 2013) du site paris-art.com de l'étonnant retour en France de l'art cinétique et en particulier de celui de Julio Le Parc : mais ce courant n'a pas été le seul, dans les années 1950-1970, à nous introduire dans un espace immersif. Il y eut aussi les espaces environnementaux, surtout en architecture ${ }^{9}$. Comme il le note avec beaucoup de pertinence, nous devons changer de cadre analytique, ce qui est évident avec une peinture de C. Coppey, même si le format du support rappelle encore celui du plan. C'est toute une esthétique qui doit revoir ses principes constituants quand elle s'émancipe de la linguistique, voire de la déconstruction derridienne, car une tache de couleur obtenue par imprégnation, frottage, pliage, n'est plus une trace. Et l'espacement entre les taches ne rend plus possible quelque chose comme une écriture. 


\section{Lumière jaune}

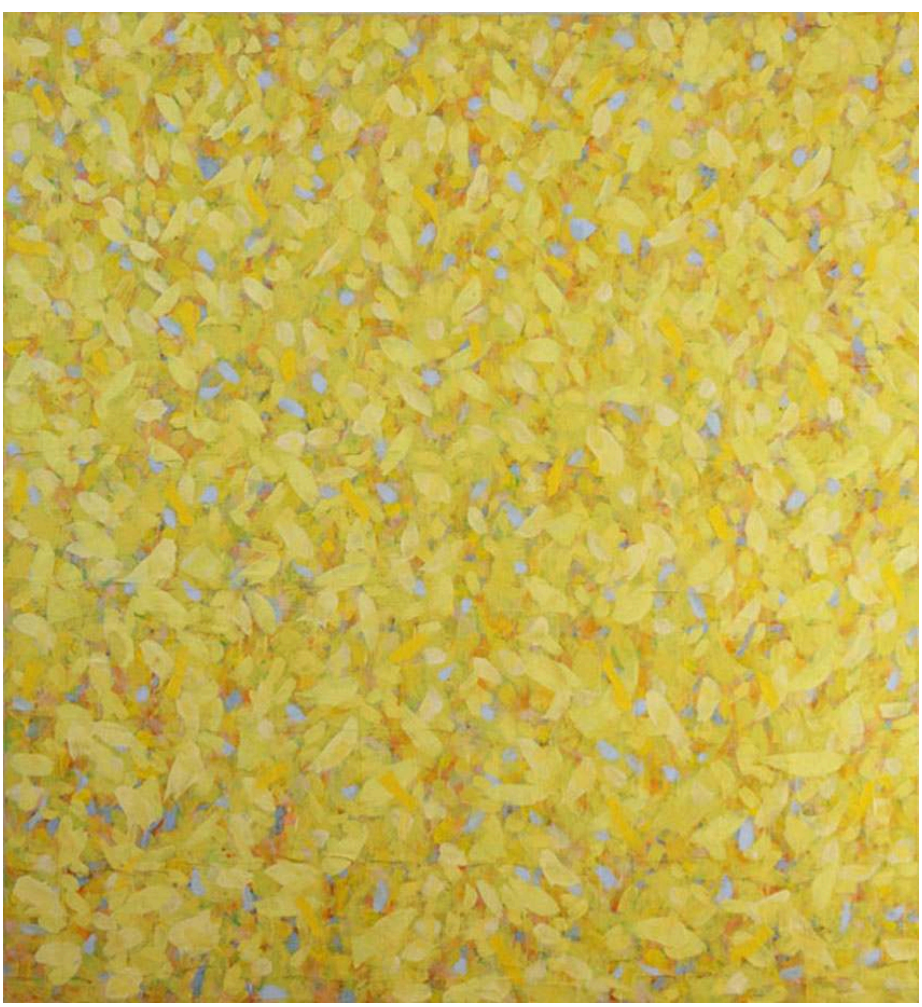

1987-1991, 163 x 151 cm, huile, vinylique, poudre de marbre et collages sur toile (c) Caroline Coppey 
Grand Jardin bleu-violet-vert

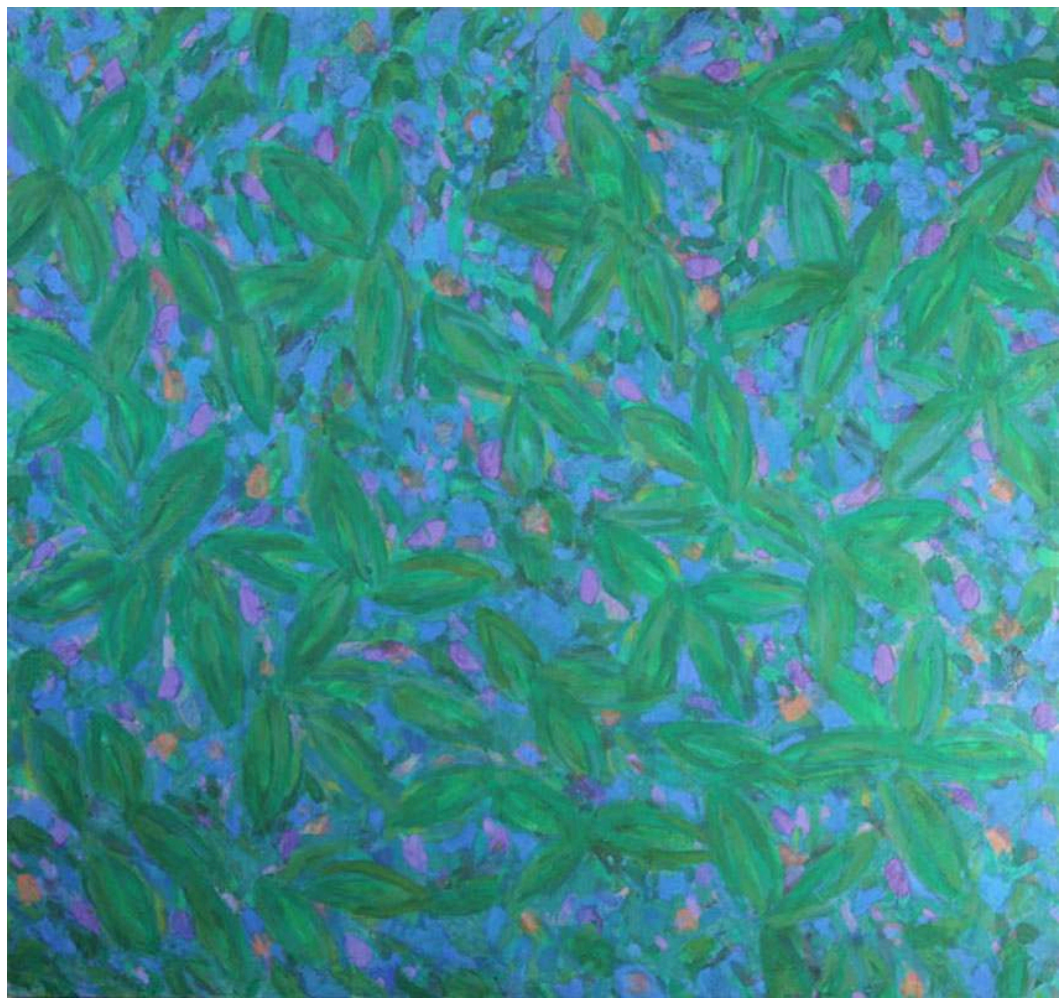

1991-1994, 183 x 195 cm, huile, vinylique, poudre de marbre et collages sur toile (C) Caroline Coppey 
Ciel 22

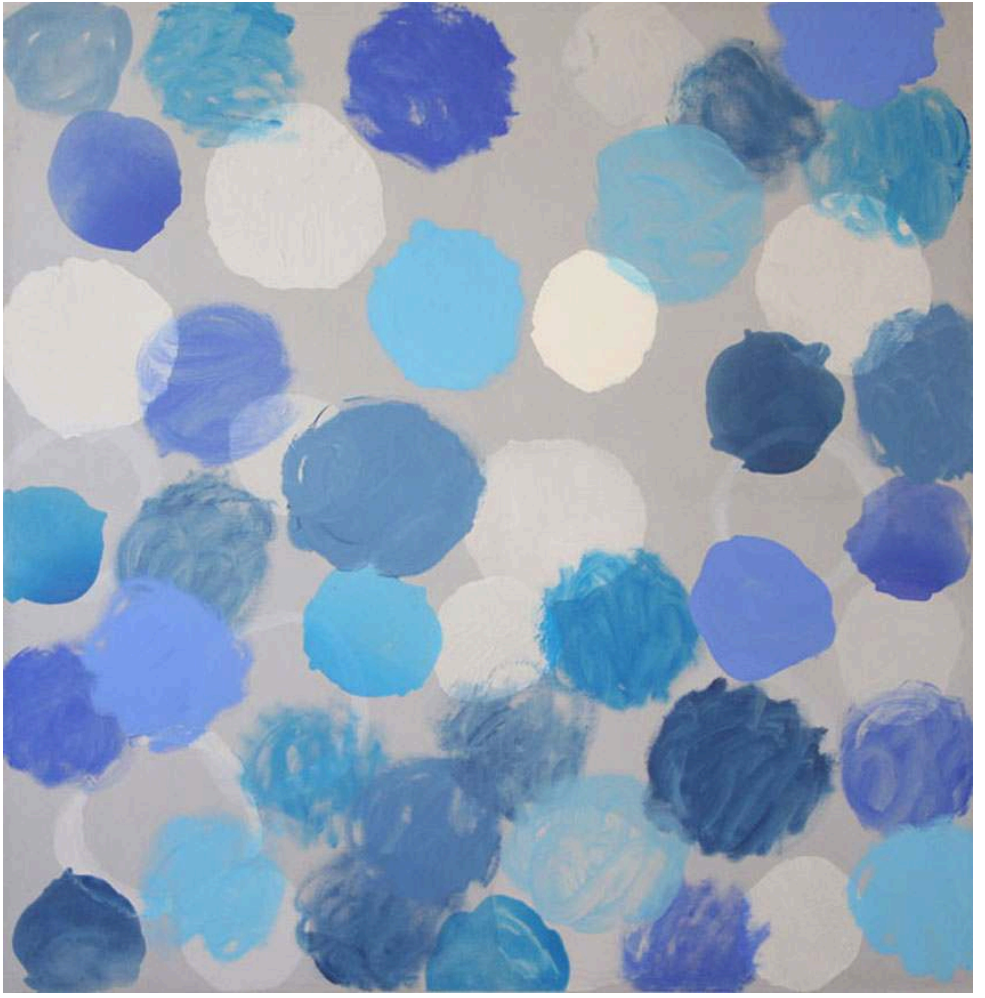

1998, $188 \times 184 \mathrm{~cm}$, huile, vinylique et collages sur toile

(c) Caroline Coppey 
P.193

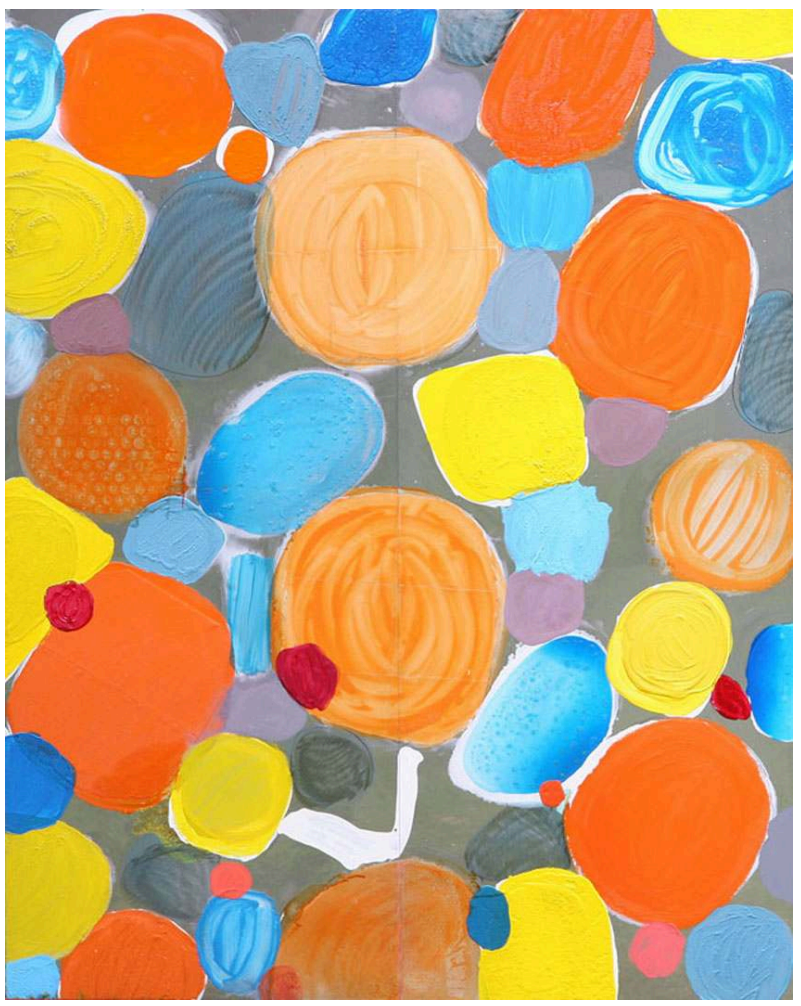

2002, $250 \times 200 \mathrm{~cm}$, acrylique, huile, poudre de marbre et collages sur inox (c) Caroline Coppey 


\section{Échantillons}

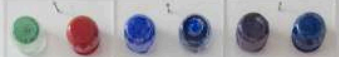

- 900 0

2 0000

Q 200

Q 0.90

- (1) 00

000

- 200

(2) 000

- 000

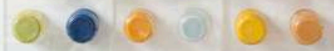

- 0000

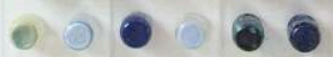

- 0 Pro

2006, $20 \times 145 \times 7 \mathrm{~cm}$ chaque module, huile, acrylique et verre sur altuglas

(c) Caroline Coppey 


\section{La Vie en Rose}

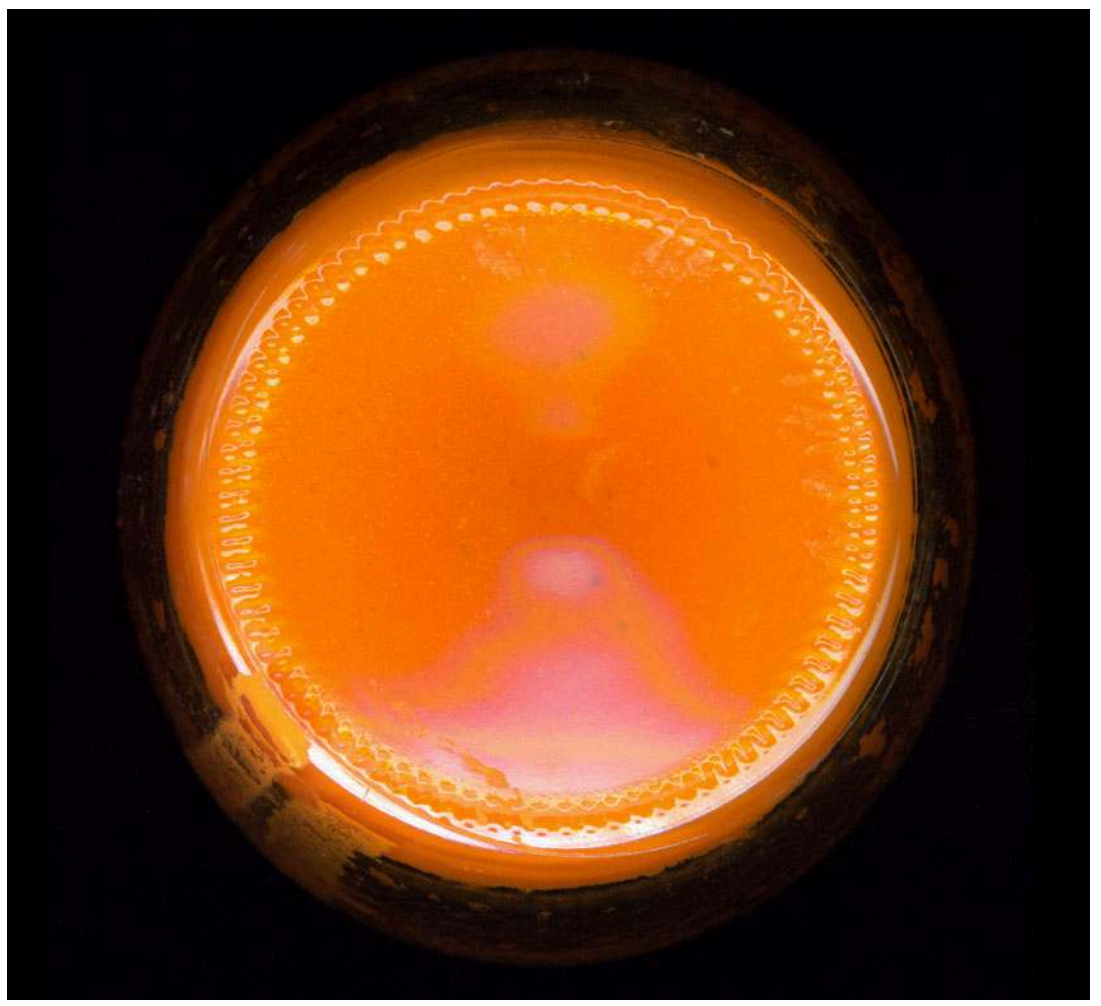

2006, Euvre numérique comportementale en vidéoprojection, conception : Caroline Coppey, développement : Atelier de création du Cube, avec le soutien de 3DVIA Virtools, Programmation: Virtools Dev 3.5, C++

(C) ART3000/LE CUBE - Caroline Coppey 


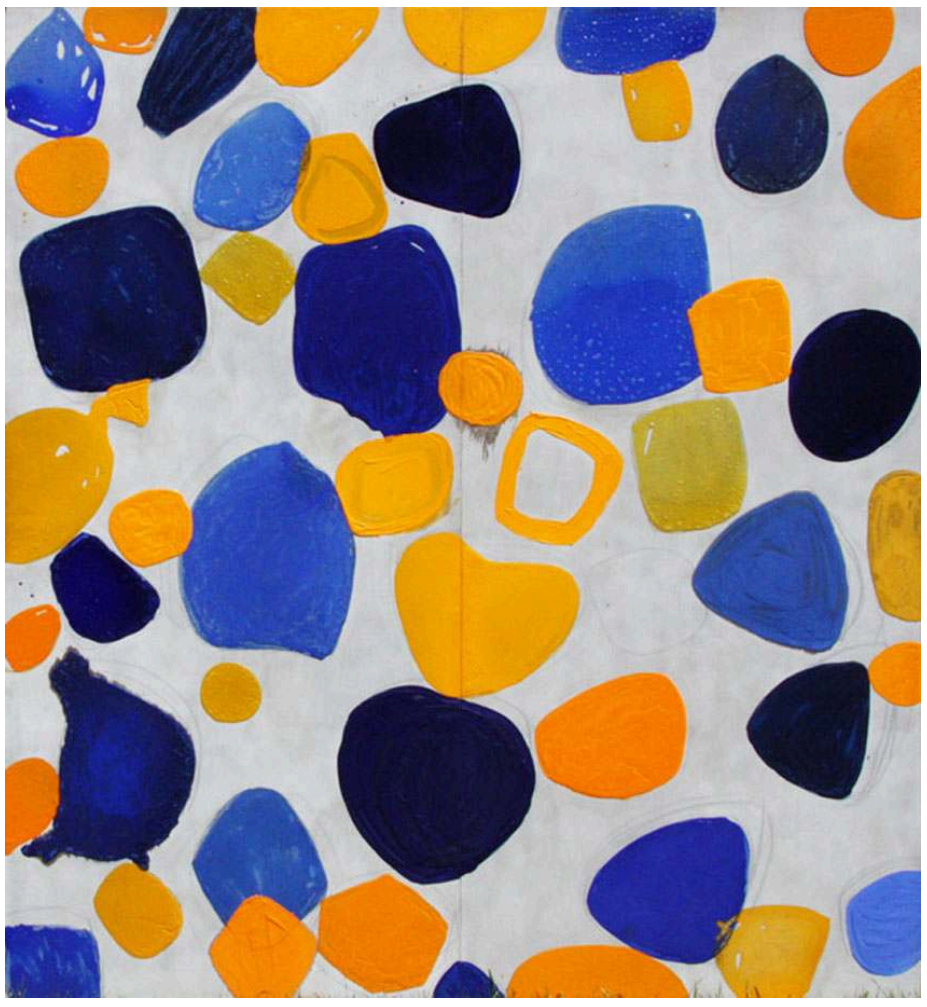

2006, $300 \times 275$ cm, huile, acrylique et caséine sur bois

(c) Caroline Coppey 
Chiffon 22

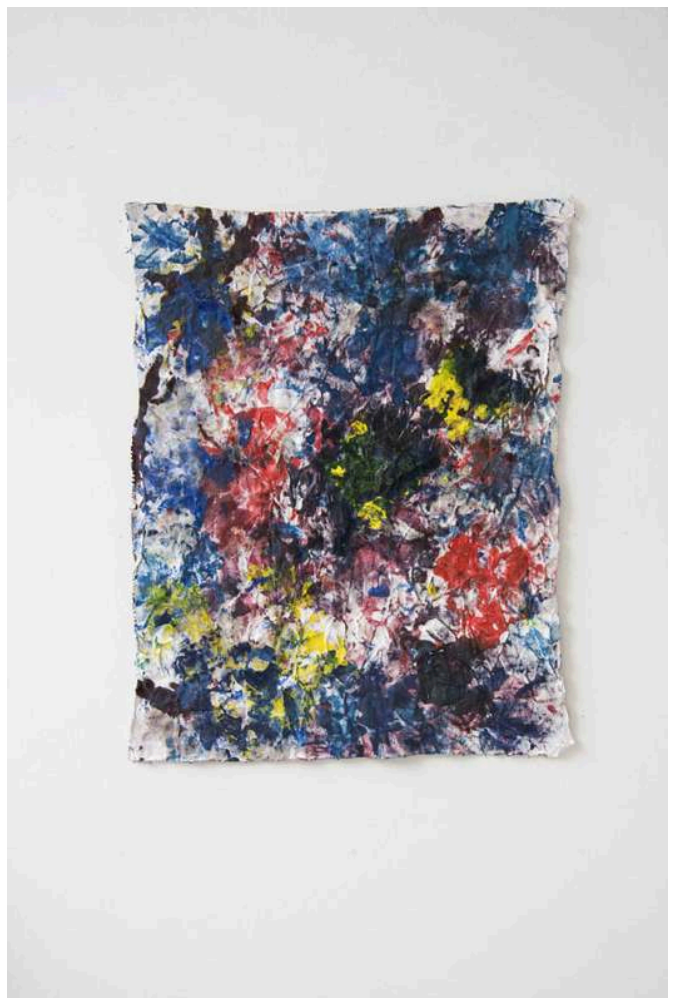

2012,64 x $50 \mathrm{~cm}$, huile et acrylique sur toile libre (c) Caroline Coppey 


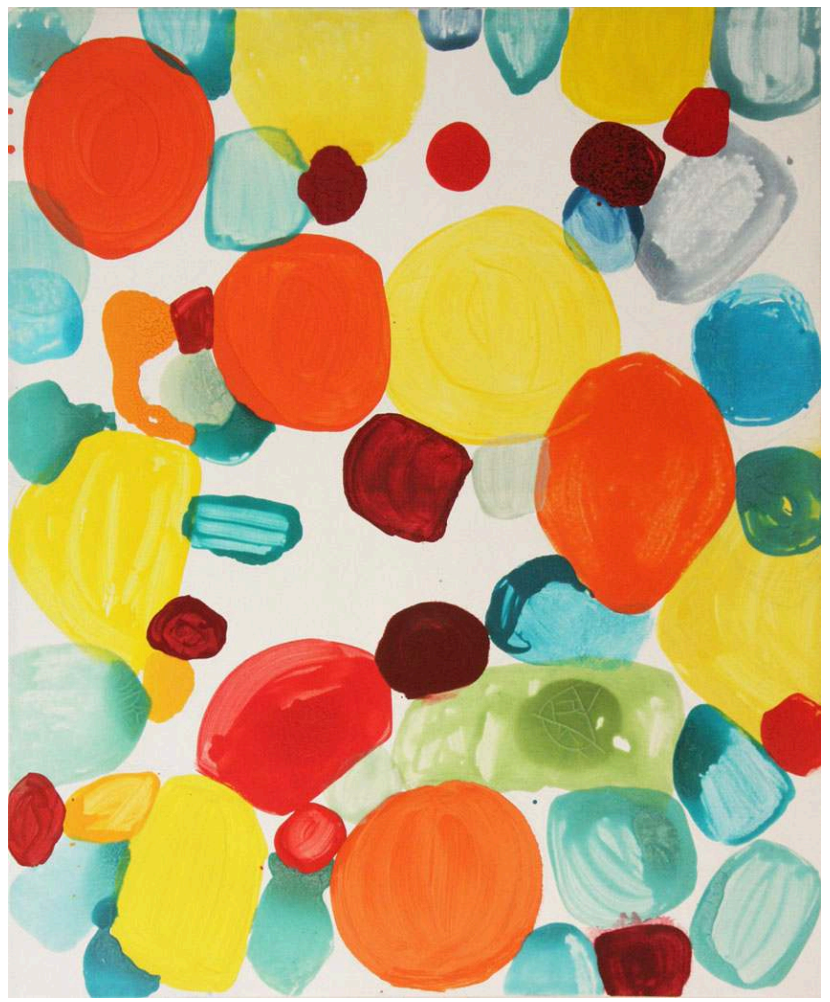

$2012,166 \times 138 \mathrm{~cm}$, huile et acrylique sur toile (c) Caroline Coppey 
Palettes 1170-1162

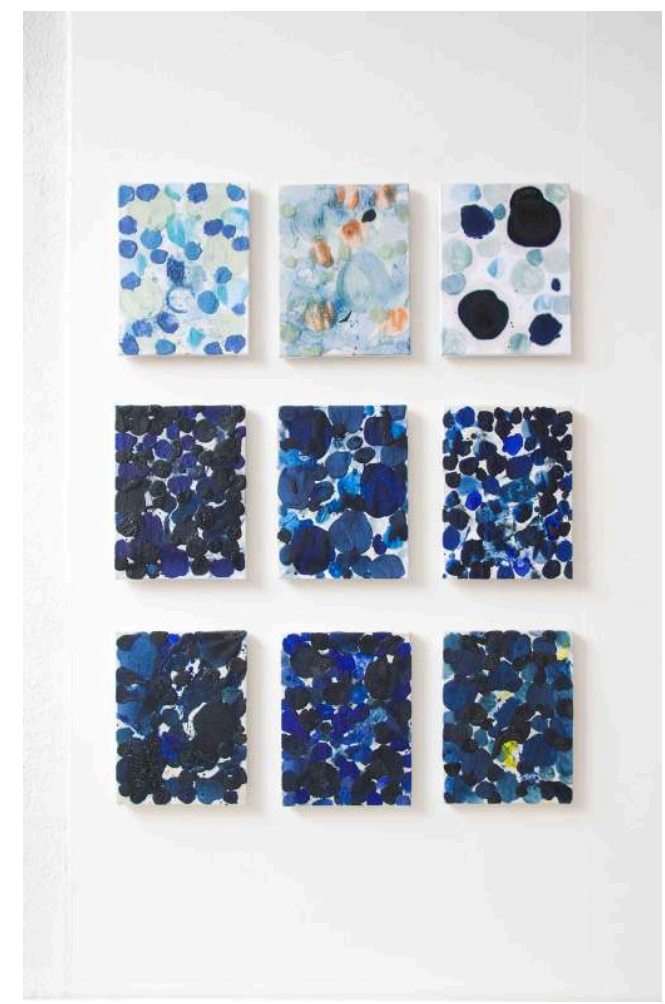

2012, 27 x 20,5 cm chaque exemplaire, huile et acrylique sur papier marouflé sur toile (c) Caroline Coppey 
Sol 26

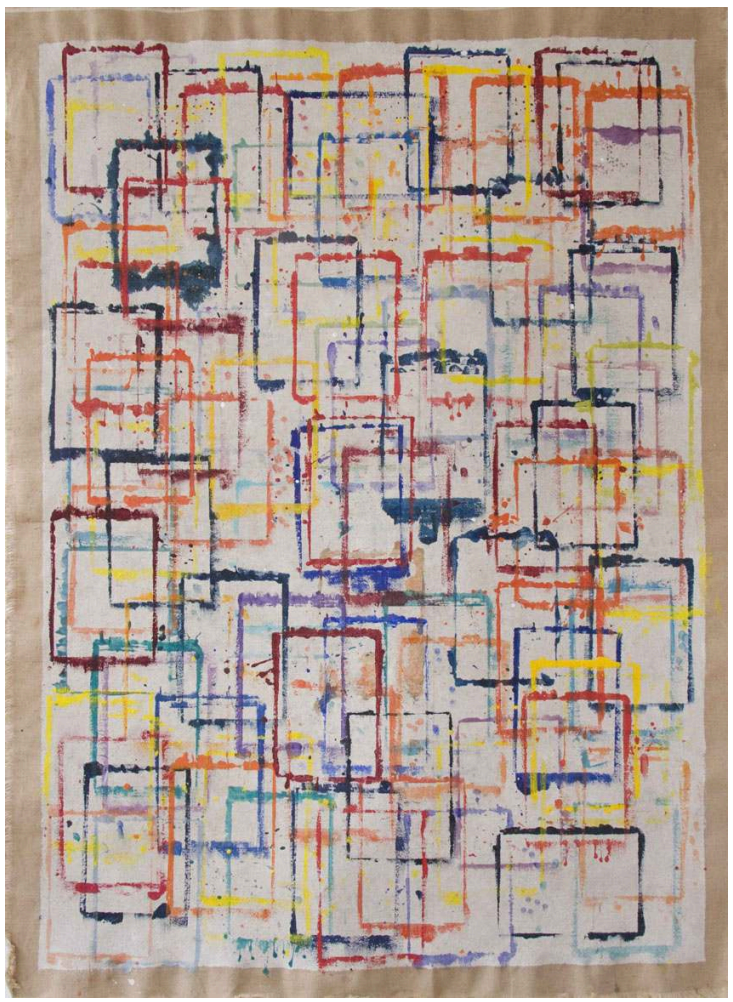

2013, $210 \times 156 \mathrm{~cm}$, huile et acrylique sur toile libre

(c) Caroline Coppey 


\section{Couleurs $n^{\circ} 9$}

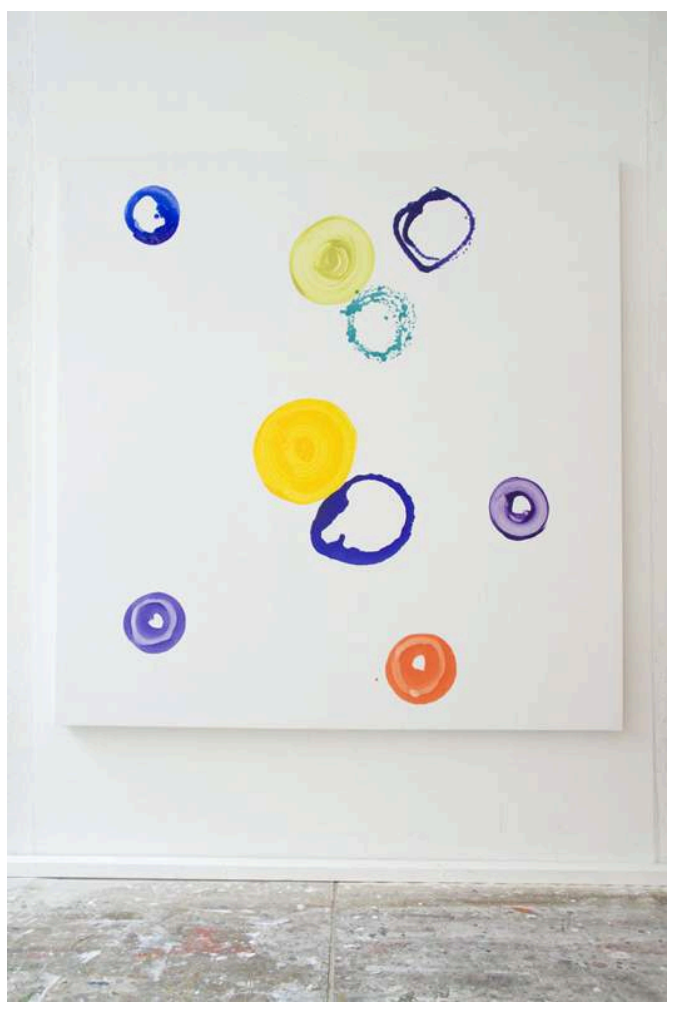

$2013,150 \times 148 \mathrm{~cm}$, huile et acrylique sur toile

(c) Caroline Coppey

\section{BIBLIOGRAPHIE}

Bellour Raymond, Le corps du cinéma. Hypnose, émotions, animalités, Paris, POL, 2009.

Crary Jonathan, L'art de l'observateur, Nîmes, J. Chambon, 1994.

Déotte Jean-Louis, Le Musée, l'origine de l'esthétique, Paris, L'Harmattan, 1993.

Déotte Jean-Louis, Walter Benjamin et la forme plastique, Paris, L'Harmattan, 2012.

Déotte Jean-Louis, « La crypte numérique », art press 2, n² 29, 13 mai 2013.

Ferry Jules, Comptes fantastiques d'Haussmann, Paris, Le Chevalier, 1868.

Krauss Rosalind, « Grilles », in L'originalité de l'avant-garde et autres mythes modernistes (1985-1993), Paris, Macula, 1993.

Lyotard Jean-François, Discours, Figure, Paris, Klincksieck, 1971.

Ponte Alessandra, Stadler Laurent, Weaver Thomas, God \& Co. François Dallegret. Beyond the Bubble, AA, 2011 


\section{NOTES}

1. Rosalind Krauss, "Grilles ", in L'originalité de l'avant-garde et autres mythes modernistes (1985-1993), Paris, Macula, 1993.

2. Jonathan Crary, L'art de l'observateur, Nîmes, J. Chambon, 1994.

3. Jean-François Lyotard, Discours, Figure, Paris, Klincksieck, 1971.

4. Ce que j'ai fait ailleurs dans Le Musée, l'origine de l'esthétique, Paris, L'Harmattan, 1993.

5. Jules Ferry, Comptes fantastiques d'Haussmann, Paris, Le Chevalier, 1868.

6. Jean-Louis Déotte, Walter Benjamin et la forme plastique, Paris, L'Harmattan, 2012.

7. C'est l'hypothèse de Raymond Bellour, Le corps du cinéma. Hypnose, émotions, animalités, Paris, POL, 2009.

8. Au sujet de l'art numérique, voir aussi mon article : «La crypte numérique », art press $2, \mathrm{n}^{\circ} 29$, 13 mai 2013.

9. Alessandra Ponte, Laurent Stadler, Thomas Weaver, God \& Co. François Dallegret. Beyond the Bubble, AA, 2011.

\section{RÉSUMÉS}

Il a fallu que la fenêtre, condition de la représentation de l'espace, se ferme, pour que l'on changeât d'ère de l'écriture des traces. On peut suivre cette évolution, puis cette rupture, en peinture, au cœur même de l'impressionnisme et en particulier chez Monet. La série des Nymphéas exposée à l'Orangerie à Paris introduit à une tout autre expérience que celle de la frontalité représentative. La focalisation sur un élément pictural n'est plus possible, le spectateur doit accepter une perception de scanning (A. Ehrenzsweig : L'ordre caché de l'art) comme celle des confins visuels dans la semi-obscurité. Or, cette époque est celle du cinéma où, contrairement à ce qu'écrit Bellour, ce n'est pas l'hypnose qui s'impose, mais des sensations proprioceptives, comme le rappelle Kracauer dans sa Théorie du film. Pour un sauvetage de la réalité.

Au même moment Aragon, dans Le Paysan de Paris, s'attachant au passage de l'Opéra, ce qui aura une très grande influence sur Benjamin, décrit l'«océan vertical» qui devient le milieu de l'humanité à venir.

\section{INDEX}

Mots-clés : espace immersif, espace de la représentation, perception de scanning, Le Paysan de Paris, Monet

\section{AUTEUR}

\section{JEAN-LOUIS DÉOTTE}

Professeur de philosophie à l'université Paris VIII (Saint-Denis) et responsable du programme de recherche « Appareils, esthétiques, arts et industries » de la MSH Paris Nord. Il dirige la collection Esthétiques chez L'Harmattan. 